\title{
Valores Lean Management como Suporte para a Gestão de Processos: uma Avaliação sobre Efetividade e Maturidade de Processos
}

\section{Lean Management Values as Support for the Process Management: an Assessment of Process Effectiveness and Maturity}

\author{
Leander Luiz Klein \\ Universidade Federal de Santa Maria - UFSM - Brasil \\ leander.klein@ufsm.br \\ ORCID: 0000-0001-6075-6107 \\ Kelmara Mendes Vieira \\ Universidade Federal de Santa Maria - UFSM - Brasil \\ kelmara@terra.com.br \\ ORCID: 0000-0002-8847-0941 \\ Thiago Schirmer Feltrin \\ Universidade Federal de Santa Maria - UFSM - Brasil \\ feltrin.schirmer@gmail.com \\ ORCID: 0000-0001-9889-9584 \\ Matheus Pissutti \\ Universidade Federal de Santa Maria - UFSM - Brasil \\ pissuttimatheus@gmail.com \\ ORCID: 0000-0001-5206-8128
}

Submetido em 15/09/2020; Aprovado em 07/11/2020.

\begin{abstract}
Resumo
Objetivo: Este trabalho tem como objetivo avaliar os valores Lean e sua relação e sua variação diante de medidas de maturidade e de efetividade da Gestão de Processos. Método: 0 método do trabalho consiste em uma pesquisa quantitativa caracterizada como survey, na qual a coleta de dados foi realizada por meio de um questionário. Obteve-se uma amostra válida de 1003 respostas da instituição estudada. A análise de dados foi realizada por meio de estatísticas descritivas, da análise fatorial exploratória (AFE), da correlação de Pearson, da análise de cluster e do teste t e do teste MannWhitney. Resultados: Os resultados permitem identificar uma relação positiva entre a melhoria contínua e o pensamento a longo prazo e a maior efetividade e maturidade de processos. Pode-se verificar, também, que a efetividade é maior no cluster em que a maturidade é maior. Contribuições: Como conclusão deste trabalho, pode-se afirmar que os valores Lean funcionam como fundamentos para a gestão baseada em processos. Os valores Lean são elementos basilares para gerar melhorias operacionais e organizacionais na condução e na realização das atividades de uma organização pública. Além disso, os resultados deste artigo contribuem para que os gestores trabalhem na consecução de parâmetros de padronização e de melhoria de processos.
\end{abstract}

Palavras-chave: Valores Lean. Gestão de processos. Efetividade de processos. Maturidade de processos. Administração pública.

\footnotetext{
Abstract

Purpose: This work aims to evaluate Lean values and their relationship and variation in view of measures of maturity and effectiveness of process management. Method: The work method consists of a quantitative survey characterized as a survey, whose data collection was carried out through a questionnaire. A valid sample of 1003 responses was obtained from the institution studied. Data analysis was performed using descriptive statistics, exploratory factor analysis, Pearson correlation, cluster analysis and $t$ and Mann-Whitney tests. Results: The results allow us to identify a positive relationship between continuous improvement and long-term thinking and the greater effectiveness
} 
and maturity of processes. It can also be seen that the effectiveness is greater in the cluster where maturity is greater. Contributions: As a conclusion of this work, it can be stated that Lean values work as the foundation for process-based management. Lean values are basic elements to generate operational and organizational improvements in the conduct and performance of the activities of a public organization. In addition, the results of this article help managers to work towards achieving standardization and process improvement parameters.

Keywords: Lean values. Process management. Process effectiveness. Process maturity. Public administration.

\section{Introdução}

As exigências da sociedade pela presteza e acuracidade cada vez maior das atividades e dos serviços públicos leva as organizações e as instituições desse setor a estabelecer meios para atingirem essas cobranças. Melhorar o antendimento, a qualidade, a presteza e a efetividade dos serviços prestados está entre as principais demandas, sempre com o máximo de transparência possível (Ferrari et al., 2018; Antony, Rodgers \& Gijo, 2016; Andrade, Rasoto \& Carvalho, 2018). Nesse sentido, a implementação e a utilização de novas práticas e modelos de gestão se faz pertinente.

Duas abordagens que propiciam eficiência e auxiliam no gerenciamento interno das práticas e dos procedimentos gerenciais têm ganhado ênfase na área pública: o Lean Management (Gupta, Sharma \& Sunder, 2016) e a Gestão de Processos (Rentes et al., 2019). O sistema Lean surgiu na indústria automobilística, mas tem ganhado grande notoriedade mundial em diversas áreas ao primar pela busca da excelência, do desperdício zero e da agregação de valor ao cliente. Já a Gestão de Processos busca continuamente o aperfeiçoamento dos procedimentos vinculados às atividades nas organizações.

A Gestão de Processos pode ser entendida como um conjunto de ações de planejamento, de direção e de avaliação das atividades da organização de forma a minimizar os conflitos interpessoais existentes e atender às expectativas, tanto interna como externa, das organizações (Oliveira, 1994). Em sua essência, a Gestão de Processos direciona a realização das atividades de maneira horizontal na estrutura hierárquica, perpassando os diversos departamentos necessários para finalização de uma determinada atividade. É uma forma de gestão oposta à tradicional estrutura funcional.

O sistema Lean, por sua vez, tem como base um conjunto de princípios de gestão, de práticas e de ferramentas que, ao serem aplicadas, auxiliam na eliminação do desperdício nas atividades operacionais e no melhor fluxo dessas atividades, tendo em vista perspectiva de valor ao cliente (Womack, Jones \& Roos, 2004; Waterman \& McCue, 2012). Para atingir esses objetivos e outros propósitos, o Lean deve ser visto como uma filosofia de gerenciamento a longo prazo que ajuda a cadeia de suprimentos da organização a identificar e a eliminar desperdícios por meio da melhoria contínua (Duarte \& Machado, 2017).

A filosofia de gestão Lean se aproxima da Gestão de Processos por compartilhar algumas características, como agregação de valor; foco na melhoria contínua; mudança de cultura organizacional; mapeamento e documentação das atividades (Rymaszewska, 2017). No entendimento de Maldonado, Leusin, Bernardes e Vaz (2020), ambas as metodologias estão baseadas em ciclos contínuos de melhoria que geram pequenas mudanças organizacionais que, por sua vez, levam a novos ciclos de melhoria. Dessa forma, a melhoria contínua e a estruturada se torna uma rotina na organização. Além disso, a implementação de valores do sistema Lean envolve um conjunto de práticas que, ao serem combinados, geram, a longo prazo, maior eficiência nos outputs organizacionais e no próprio gerenciamento de processos (Bhasin \& Burcher, 2006).

No entanto, a relação dos fundamentos do sistema Lean com a maturidade e a efetividade de processos ainda não está claramente compreendida. Mesmo que o sistema Lean tenha sido definido como um conjunto de princípios e de técnicas para aumentar a eficiência de processos (Shah \& Ward, 2003) e que ambas as abordagens compartilham elementos semelhantes, não há clareza na literatura sobre essa relação no setor de serviços. 0 que dificulta isso é a maior variabilidade existente no referido setor, o que gera mais dificuldade na definição de termos e na padronização de procedimentos (Cavdur et al., 2019). Dave (2017) também argumenta que, além das interconexões entre pessoas e processos e tecnologia, o planejamento e a análise de elementos basilares são outros fatores críticos para as iniciativas de Gestão de Processos. Diante disso, entender as diferenças de percepções e a relação entre os valores da filosofia Lean com medidas de outputs de processos se torna válido tanto 
na teoria quanto na prática. 0 problema de pesquisa deste trabalho é em que medida os valores Lean se associam e variam diante das medidas de efetividade e de maturidade de processos?

Hernaus, Vuksic e Štemberger (2016) afirmam que o Lean Management é um reforço para iniciativas focadas em processos nas organizações que visam aumentar sua eficiência e eficácia. 0 uso de práticas e de valores Lean pode melhorar também a produtividade e a eficiência de processos organizacionais (Aqlan \& Al-Fandi, 2018). Considerando isso, o presente artigo tem como objetivo avaliar os valores Lean e sua relação e variação diante de medidas de maturidade e de efetividade da Gestão de Processos.

0 estudo proposto neste trabalho se torna relevante na medida em que os valores Lean podem gerar uma melhoria na maneira que os processos são organizados e efetuados, uma vez que se referem a um empreendimento contínuo que visa a eliminação de desperdícios baseado na criação de valor para os usuários (Bashin \& Burcher, 2006; Modig \& Ảhlström, 2012). Nesse sentido, a realização dessa pesquisa gera contribuições para a literatura ao estreitar as lacunas de entendimento acerca dessas duas temáticas, principalmente no setor de serviços públicos. Além das contribuições teóricas, este estudo tem como inovação a ampliação dos limites de aplicação e de avaliação das práticas Lean para o âmbito das organizações públicas. O entendimento de como os valores do sistema Lean estão associados à Gestão de Processos permite que os gestores tomem decisões mais precisas sobre as atividades realizadas.

\section{Referencial Teórico}

\subsection{Valores Lean Management}

O Lean Management pode ser conceituado como uma filosofia de gestão que visa identificar e eliminar desperdícios em toda a cadeia de valor de negócios e não apenas internamente na organização (Scherrer-Rathje, Boyle \& Deflorin, 2009). A eliminação de desperdícios é concebida para muitos como um princípio ou um valor central da gestão Lean (Womack, Jones \& Roos, 2004), sendo esse um objetivo permanente das organizações que adotam e buscam implementar essa filosofia de gestão. Alinhado a esse objetivo, está a prática de melhoria contínua dos procedimentos, seja de processos internos ou externos, seja de atividades operacionais ou gerenciais. Para se tornar efetiva, a ação de melhoria contínua requer a participação de todos na organização (Uhrin, Bruque-Cámara \& MoyanoFuentes, 2017).

Alinhado ao supracitado, um dos principais objetivos do sistema Lean é produzir produtos ou serviços de maior qualidade ao menor custo e no menor tempo, com menores perdas e com menor variabilidade aos clientes (Shah \& Ward, 2007). Para atingir esses objetivos, o Lean pode ser visto como uma filosofia de gerenciamento que envolve a utilização de várias ferramentas e práticas (Waterman \& McCue, 2012).

Paralelamente, estudos surgiram para descrever elementos que foram definidos como valores do sistema Lean. Não há um claro posicionamento na literatura sobre o assunto, nem uma distinção nítida do que são práticas e do que são valores Lean. Nesse sentido, Shah e Ward (2003) destacam a importância da visão sistêmica para o entendimento geral da organização e de como as atividades se interligam para o alcance dos objetivos da organização. Liker (2004), por sua vez, destaca o pensamento a longo prazo como um dos princípios basilares da filosofia Lean, mesmo que algumas atividades acarretem em perdas a curto prazo. Madsen et al. (2019) corroboram ao afirmarem que o pensamento a longo prazo intensifica e torna as empresas mais eficientes para implementarem processos e outros valores e outras práticas da organização enxuta.

Quando se pesquisa sobre as práticas do sistema Lean, o foco no público-alvo e na criação de valor aos usuários (clientes) é tida como o parâmetro para estabelecer melhorias contínuas e a redução de desperdícios. Para Shamah (2013), a perspectiva de valor para o público-alvo jamais deve ser desconsiderada e é, essencialmente, a capacidade de fornecer produtos ou serviços conforme requisitados pelo usuário final do produto ou do processo.

Nesse contexto, o apoio dos líderes e da alta gestão das organizações é fundamental, uma vez que direciona e dá suporte para os esforços de realização e de consecução das outras práticas e dos outros valores, além de aproximar os diferentes níveis dentro da organização. A liderança precisa ser enxuta em todos os seus níveis, mesmo onde a ordem de hierarquia é ressaltada, pois isso acaba gerando uma interação e uma intenção maior de todos colaboradores em melhorar (Siedel, Rust, Goth, 
Krüger \& Heidenfelder, 2019).

Os apontamentos supracitados são apenas a descrição de algumas das principais práticas ou valores Lean, conforme o entendimento de diferentes estudiosos. Na Tabela 1, apresenta-se um compilado de principais práticas e valores Lean Management descritos na literatura sobre o assunto.

Tabela 1 - Principais práticas e valores Lean citadas na literatura

\begin{tabular}{|c|c|c|c|c|c|c|c|c|c|c|c|c|c|c|}
\hline \multirow{2}{*}{ Práticas Lean } & \multicolumn{14}{|c|}{ Estudos } \\
\hline & 1 & 2 & 3 & 4 & 5 & 6 & 7 & 8 & 9 & 10 & 11 & 12 & 13 & 14 \\
\hline Visão sistêmica & $\mathrm{x}$ & & & & $\mathrm{x}$ & & & & & & & & & \\
\hline Pensamento a longo prazo & & $\mathrm{x}$ & $\mathrm{x}$ & & $\mathrm{x}$ & & & & & $\mathrm{x}$ & & & $\mathrm{x}$ & \\
\hline Eliminação de desperdícios & $\mathrm{x}$ & $\mathrm{x}$ & & & $\mathrm{x}$ & & & $\mathrm{x}$ & $\mathrm{x}$ & & & & & \\
\hline Melhoria contínua & $\mathrm{x}$ & $\mathrm{x}$ & $\mathrm{x}$ & & $\mathrm{x}$ & $\mathrm{x}$ & & & $\mathrm{x}$ & $\mathrm{x}$ & & & & \\
\hline Foco no público-alvo & $\mathrm{x}$ & & $\mathrm{x}$ & $\mathrm{x}$ & $\mathrm{x}$ & & & & $\mathrm{x}$ & & & & $\mathrm{x}$ & \\
\hline Apoio da liderança & & & $\mathrm{x}$ & & $\mathrm{x}$ & & & & & & & & & $\mathbf{x}$ \\
\hline Envolvimento do fornecedor & & $\mathrm{x}$ & $\mathrm{x}$ & & $\mathrm{x}$ & & $\mathrm{x}$ & & & & $\mathrm{x}$ & & & \\
\hline Gestão de qualidade & $\mathrm{x}$ & & & & & & & & $\mathrm{x}$ & & & $\mathrm{x}$ & & \\
\hline
\end{tabular}

Fonte: Elaborada pelos autores.

Estudos nas colunas: 1 - Womack, Jones e Roos (2004), 2 - Liker (2004), 3 - Bhasin e Burcher (2006), 4 - Gudem, Steinert, Welo e Leifer (2013), 5 - Ingelsson e Mårtensson (2014), 6 - Narayanamurthy e Gurumurthy (2016), 7 - Marodin, Tortorella, Frank e Godinho Filho (2017), 8 - Johansson e Osterman (2017), 9 - Wickramasinghe e Wickramasinghe (2017), 10 - Uhrin et al. (2017), 11 - Gómez-Luciano, Domínguez, González-Andrés e De Meneses (2018), 12 - Ghobakhloo, Fathi, Fontes e Ching (2018), 13 - Madsen et al. (2019), 14 - Siedel et al. (2019).

De maneira a definir os valores Lean utilizados neste trabalho, elaborou-se a Tabela 2, com as definições e as explicações destes, além dos autores de referência.

Tabela 2 - Definição de valores Lean utilizados no trabalho

\begin{tabular}{|c|c|c|}
\hline Valor Lean & Definição/explicação & Referências \\
\hline $\begin{array}{c}\text { Visão } \\
\text { sistêmica }\end{array}$ & $\begin{array}{l}\text { Considera o entendimento de como o trabalho de uma pessoa está ligado a outras } \\
\text { partes da organização e como ele se encaixa nos objetivos gerais da organização. }\end{array}$ & $\begin{array}{l}\text { Ingelsson e Martens- } \\
\text { son (2014). }\end{array}$ \\
\hline $\begin{array}{l}\text { Pensamento a } \\
\text { longo prazo }\end{array}$ & $\begin{array}{l}\text { A tomada de decisões é realizada em uma visão de longo prazo articulada e plane- } \\
\text { jada, mesmo se elas tiverem como contrapartida algo não benéfico financeira- } \\
\text { mente no curto prazo. Envolve uma visão comum de como melhorar as atividades } \\
\text { do setor. }\end{array}$ & $\begin{array}{l}\text { Liker (2004); Bhasin } \\
\text { e Burcher (2006). }\end{array}$ \\
\hline $\begin{array}{l}\text { Eliminação de } \\
\text { desperdícios }\end{array}$ & $\begin{array}{l}\text { O desperdício é compreendido como qualquer forma de ação ou tarefa desneces- } \\
\text { sária que, além de poder incidir no aumento dos custos de produção, não gera } \\
\text { valor ao produto ou ao serviço em si. Sua eliminação envolve as ações de identifi- } \\
\text { car, de reduzir ou de cortar os desperdícios o mais rápido possível, e diz respeito } \\
\text { a todos os tipos de desperdícios definidos na filosofia Lean. }\end{array}$ & $\begin{array}{l}\text { Womack, Jones e } \\
\text { Roos (2004); Jo- } \\
\text { hansson e Osterman } \\
\text { (2017); Hussain e } \\
\text { Malik, 2016). }\end{array}$ \\
\hline $\begin{array}{l}\text { Melhoria } \\
\text { contínua }\end{array}$ & $\begin{array}{l}\text { Pode ser definida como empenho contínuo dos funcionários na efetivação de } \\
\text { mudanças, mesmo que pequenas, nas atividades para que gerem transformações } \\
\text { de melhoria incrementais. Isso envolve a disponibilização de tempo e uma ma- } \\
\text { neira padronizada de implementar essas melhorias no trabalho diário. }\end{array}$ & $\begin{array}{l}\text { Uhrin et al. (2017); } \\
\text { Aka, Isah, Eze e } \\
\text { Timileyin (2019). }\end{array}$ \\
\hline $\begin{array}{l}\text { Foco no } \\
\text { público-alvo }\end{array}$ & $\begin{array}{l}\text { Definido a partir da noção de valor ao usuário, ou seja, algo para o qual o "cliente" } \\
\text { está disposto a pagar. Não possui desperdícios associados ao produto ou ao ser- } \\
\text { viço, ao menos pelos olhos do usuário. Envolve conhecer quem é esse usuário e } \\
\text { saber suas necessidades. }\end{array}$ & $\begin{array}{l}\text { Madsen et al. (2019), } \\
\text { Gudem et al. (2013). }\end{array}$ \\
\hline $\begin{array}{l}\text { Apoio da } \\
\text { liderança }\end{array}$ & $\begin{array}{l}\text { Refere-se às atitudes e aos comportamentos dos gestores e dos líderes que reper- } \\
\text { cutem nas ações do restante dos funcionários na organização. Envolve as respon- } \\
\text { sabilidades, o apoio e o estímulo, a comunicação e a forma de trabalho direciona- } \\
\text { da pelas lideranças (chefias e gestores). }\end{array}$ & $\begin{array}{l}\text { Ingelsson e } \\
\text { Mårtensson (2014); } \\
\text { Alefari, Salonitis e Xu } \\
\text { (2017). }\end{array}$ \\
\hline
\end{tabular}

Fonte: Elaborada pelos autores.

\subsection{Gestão de Processos}

A Gestão de Processos, alicerçada nos princípios do BPM (Business Process Management), vem sendo adotada por muitas organizações como uma nova forma organizacional para alcançar eficiência, eficácia e desempenho organizacional em suas atividades (Castro, Dresch \& Veit, 2020). Adotar a prática de Gestão de Processos auxilia na geração de valor aos seus clientes e produz efeitos positivos que aumentam o desempenho dos próprios processos, geram satisfação dos usuários do sistema e diminuem o tempo de produção e os custos (Kohlbacher, 2010).

o gerenciamento de processos é uma abordagem utilizada para analisar e melhorar constantemente os processos de uma organização (Zairi, 1997) e pode ser considerado como um conjunto de métodos, de técnicas e de ferramentas para descobrir, para redesenhar, para analisar, para monitorar e para executar os processos das organizações (Dumas, La Rosa, Mendling \& Reijers, 
2013; Castro et al., 2020). 0 estudo dos processos, no entendimento de Kohlbacher (2010), é de suma importância para o desenvolvimento de soluções que permeiam, em sua maioria, o aumento da velocidade e a melhoria na execução das atividades; a adaptação às mudanças; a satisfação dos clientes; a redução de custos; e o melhor: a compreensão das atividades presentes na organização.

Além disso, outro elemento que pode ser melhorado pela Gestão de Processos é a satisfação dos clientes. Klun e Trkman (2018) argumentam que a Gestão de Processos refere-se a uma técnica que permite avaliar, analisar e melhorar o desempenho dos processos organizacionais que impactam de forma direta na satisfação dos clientes. Ou seja, o sucesso da satisfação dos clientes está estreitamente interligado à correta execução dos processos da organização (Carballo-Mendivil, Arellano-Gonzalez \& Rios-Vazquez, 2018).

A maior parte dos modelos de Gestão de Processos sugere que sejam compreendidos os processos atuais que a organização possui para que, dessa forma, possam ser identificados aqueles que estão em desequilíbrio com o restante da organização. A partir dessa identificação, as organizações podem se organizar e implementar melhorias para alcançar maiores níveis de efetividade nas suas áreas funcionais. A efetividade dos processos pode ser definida como uma medida de saída para o sucesso dos processos, por avaliar os mais bem sucedidos, demonstrando outputs com custos mais baixos e também tempos de ciclos mais rápidos (Schymik, Kulkarni \& Freeze, 2007). Na percepção de Kohlbacher (2010), diferentes mecanismos de gerenciamento de processos podem ser aplicados para melhorar o desempenho dos processos organizacionais em termos de efetividade. Neste trabalho, temse o pressuposto de que os valores Lean sirvam de base para essa melhoria.

Estreitamente alinhada à efetividade de processos está a maturidade de processos, uma vez que esta pode ser entendida como o grau de acuracidade de processos de uma organização com base em competências e habilidades dos colaboradores para executá-los (Krukowski \& Raczynska, 2019). Os modelos de maturidade de processos constituem-se em ferramentas evolutivas que servem para avaliar e melhorar as capacidades e os recursos das organizações com o intuito de alcançar a excelência nos processos (Van Looy, De Backer \& Poels, 2011). Ou seja, quanto maior for o nível da maturidade, maior a possibilidade da empresa alcançar a excelência e a efetividade organizacional.

Diante dos apontamentos teóricos realizados, pode-se perceber a proximidade entre as abordagens Lean e Gestão de Processos. Ambas pressupõem uma nova forma gerencial baseada na busca de melhorias contínuas por meio de mudanças menores que devem ocorrer regularmente na organização, e não necessariamente ser uma mudança radical e revolucionária (Rymaszewska, 2017). Essa melhoria contínua dentro da organização, seja por meio de atividades internas ou externas, ampara o desempenho dos processos (Uhrin et al., 2017; Kohlbacher, 2010).

A descrição e a definição teórica de valores Lean, Gestão de Processos e maturidade e efetividade de processos mostra que existe uma aproximação (associação) entre as duas abordagens de gestão, assim como apresentado no trabalho de Maldonado et al. (2020). Baseado nisso, foi elaborada a seguinte hipótese de pesquisa:

- Hipótese 1: Há uma associação positiva entre a percepção sobre os valores Lean e a maturidade e efetividade de processos.

Além disso, a partir do referencial teórico estabelecido, busca-se averiguar a hipótese de que, na instituição estudada, existem grupos de servidores com percepções homogêneas no grupo, mas, em média, diferentes entre os grupos. Por isso, estabeleceu-se a segunda e a terceira hipóteses de pesquisa, que são:

- Hipótese 2: 0 grupo com melhor percepção de maturidade e efetividade de processos também apresenta, em média, melhores percepções quanto aos valores Lean avaliados.

- Hipótese 3: 0 grupo com melhor percepção de maturidade de processos também apresenta, em média, melhores percepções quanto à efetividade de processos.

\section{Método da Pesquisa}

Para alcançar o objetivo deste trabalho, realizou-se uma pesquisa quantitativa do tipo survey, pois esse tipo de pesquisa permite reunir uma grande quantidade de informações acerca de uma determinada população em que as perguntas possuem alternativas de respostas delimitadas (Hair, Black, Babin, Anderson \& Tatham, 2005).

Para a consecução da coleta de dados foi elaborado um questionário estruturado composto por 3 
seções principais, conforme demonstrado na Tabela 3. Nela, são exibidos os constructos que compunham o instrumento de pesquisa, o número de questões e os estudos de onde elas foram retiradas. Como medida de mensuração das respostas, utilizou-se uma escala do tipo Likert de 10 pontos, sendo 1 (baixa concordância com o conteúdo da pergunta) e 10 (alta concordância com o conteúdo da pergunta).

Tabela 3 - Resumo das questões sobre o questionário da pesquisa

\begin{tabular}{|c|c|c|c|}
\hline Seção & Constructo & Questões & Referências \\
\hline \multirow{6}{*}{ Seção 1 - Valores Lean } & Visão sistêmica & 03 & \multirow{6}{*}{ Ingelsson e Mårtensson (2014) } \\
\hline & Pensamento a longo prazo & 03 & \\
\hline & Eliminação de desperdícios & 06 & \\
\hline & Melhoria contínua & 04 & \\
\hline & Foco no público-alvo & 03 & \\
\hline & Apoio da liderança & 05 & \\
\hline \multirow{2}{*}{$\begin{array}{l}\text { Seção } 2 \text { - Maturidade e } \\
\text { efetividade de processos }\end{array}$} & Maturidade & 08 & AlShathry (2016) \\
\hline & Efetividade & 06 & $\begin{array}{c}\text { Isik, Mertens e Van den Bergh } \\
\text { (2013) }\end{array}$ \\
\hline Seção 3 & Perfil & 07 & Elaboradas pelos autores \\
\hline
\end{tabular}

Fonte: Elaborada pelos autores.

As perguntas (itens) do questionário (Tabela 5) foram traduzidas por um dos pesquisadores, o qual possui fluência de leitura na língua inglesa. Após isso, foi realizada uma validação de conteúdo das perguntas do instrumento com dois professores (um com experiência na elaboração de questionários on-line; outro, pesquisador da área de Lean) e dois policiais federais. 0 objetivo desse procedimento foi avaliar o entendimento e a adequabilidade das questões e corrigir as questões que pudessem ter interpretações dúbias ou deixar algum respondente constrangido. Depois disso, foi feita uma nova averiguação pelo pesquisador, que traduziu o instrumento para verficar se o sentido das perguntas não foi alterado com as adaptações.

Concluídas as adaptações no instrumento, a coleta de dados foi realizada de forma on-line, com a utilização da ferramenta "Questionário Eletrônico" do Google Forms. Dessa forma, foi elaborada uma versão on-line desse questionário e enviado um convite por e-mail e para os grupos de Whatsapp dos integrantes da população alvo da pesquisa. Considera-se essas duas formas de divulgação complementares, pois o acesso de uma não limita ou restringe o acesso a outra.

0 estudo foi realizado na instituição Polícia Federal (PF) do Brasil. A população deste estudo consiste nos servidores ativos da PF. Especificamente, foram contactados os servidores administrativos ativos filiados à Federação Nacional dos Policiais Federais (FENAPEF). 0 total de servidores ativos, em março de 2020, era de 8.491. A escolha por essa entidade federativa pública teve dois motivos: (1) a possibilidade de acesso à população alvo para realização da pesquisa da forma como ela foi feita e (2) o fato de a entidade aderir à abordagem gerencial baseada em processos. Conforme as informações obtidas na entidade, a PF trabalha na identificação, no mapeamento, no diagnóstico e no aperfeiçoamento das atividades e das diligências (processos), fornecendo aos servidores envolvidos os treinamentos e as capacitações adequadas (Polícia Federal, 2014). A coleta de dados ocorreu nos meses de abril, maio e junho de 2020 e obteve-se amostra válida de 1003 respondentes.

Os dados obtidos foram analisados com a utilização do Software SPSS ${ }^{20}$. Como procedimentos de análise dos dados efetuou-se, primeiramente, o cálculo de estatísticas descritivas para a análise do perfil dos respondentes. Em seguida, utilizou-se a técnica de análise fatorial exploratória (AFE) para congregação dos dados e da obtenção de fatores para análise. Seguindo os postulados de Hair et al. (2005), verificou-se, primeiramente, a fatorabilidade dos dados (para todos os itens) por meio do teste de esfericidade de Bartlett e o cálculo do índice de Kaiser-Meyer-Olkin (KMO). Para Pestana e Gageiro (2008), esses dois testes servem para identificar a qualidade das correlações entre as variáveis e verificar a fatorabilidade dos dados. Em seguida, as comunalidades dos itens foram avaliadas, excluindo aqueles que apresentaram valores menores que 0,5 para essa medida (Hair et al., 2005). Por fim, a determinação do número de fatores foi feita a partir do critério de estimação Eigenvalue, e como método rotacional utilizou-se a rotação Varimax. Conforme Hair et al. (2005), a AFE realiza a exploração dos dados e, a partir disso, fornece as informações sobre a quantidade de fatores que é necessário para melhor representar os dados a partir de uma estimativa de carga fatorial.

Após efetuar a análise dos fatores, foi verificada a associação entre eles por meio da análise de 
correlação de Pearson, a qual envolveu a verificação da "força" de associação entre dois fatores. Para tal, considerou-se a convenção de Pestana e Gageiro (2008) que delimita o coeficiente de correlação em: abaixo de 0,2, muito baixo; entre 0,2 e 0,39, baixo; entre 0,4 e 0,69, moderado; entre 0,7 e 0,89, alto; e acima de 0,9 , muito alto.

Por fim, foram efetuadas duas análises de clusters, que tem como finalidade agregar elementos em vista de características comuns que eles possuem (Hair et al., 2005) para separar a amostra em grupos. 0 procedimento para criação dos clusters foi o seguinte: primeiramente, analisou-se o dendograma para definir a quantidade de clusters a serem formados; em seguida, utilizou-se o método hierárquico de Ward para formar os clusters por ser um dos mais consistentes para escalas intervalares (Hair et al., 2009). A primeira análise de cluster utilizou os fatores de maturidade e de efetividade de processos para formação dos clusters. A partir dos clusters formados, efetuou-se o teste t e o teste Mann-Whitney para verificar se havia diferença estatística quanto à percepção média de aplicação dos valores Lean. A segunda análise de cluster foi feita com base em todos os itens que compunham o fator "Maturidade de processos". A partir dos clusters obtidos, foram efetuados novamente o teste t e o teste Mann-Whitney para averiguar se a média de efetividade de processos era estatisticamente diferente nos dois grupos.

\section{Resultados da Pesquisa}

\subsection{Características e perfil da amostra}

A amostra investigada, conforme referido anteriormente, foi composta por 1003 respondentes. Foram observados alguns aspectos que pudessem especificar e caracterizar sumariamente a amostra investigada. Esses aspectos são apresentados na Tabela 4.

Tabela 4 - Perfil dos respondentes

\begin{tabular}{|c|c|c|}
\hline Variável & Alternativa & Percentual \\
\hline \multirow{2}{*}{ Sexo } & Masculino & $61 \%$ \\
\hline & Feminino & $39 \%$ \\
\hline \multirow{4}{*}{ Faixa etária } & De 26 a 36 anos & $28,4 \%$ \\
\hline & De 37 a 47 anos & $49,5 \%$ \\
\hline & De 48 a 58 anos & $18,2 \%$ \\
\hline & Acima de 58 anos & $3,90 \%$ \\
\hline \multirow{4}{*}{ Estado civil } & Solteiro (a) & $22,3 \%$ \\
\hline & Casado (a) ou relação estável & $55,9 \%$ \\
\hline & Separado (a) & $20,1 \%$ \\
\hline & Viúvo (a) & $1,6 \%$ \\
\hline \multirow{4}{*}{ Nível de escolaridade } & Ensino médio & $9,2 \%$ \\
\hline & Graduado (a) & $72,8 \%$ \\
\hline & Especialista & $16,6 \%$ \\
\hline & Mestre (a) ou Doutor (a) & $1,5 \%$ \\
\hline \multirow{2}{*}{ Chefe de algum setor } & Sim & $11,7 \%$ \\
\hline & Não & $88,3 \%$ \\
\hline \multirow{3}{*}{$\begin{array}{l}\text { Tempo de serviço na Polícia Fede- } \\
\text { ral }\end{array}$} & Até 5 anos & $7,2 \%$ \\
\hline & 5 a 10 anos & $41,9 \%$ \\
\hline & Mais do que 10 anos & $50,9 \%$ \\
\hline \multirow[b]{2}{*}{$\begin{array}{l}\text { Principal motivo da padronização } \\
\text { de processos }\end{array}$} & Interesse do próprio setor. & $52,4 \%$ \\
\hline & $\begin{array}{l}\text { Haver uma política da PF incentivando a Gestão de Pro- } \\
\text { cessos }\end{array}$ & $47,6 \%$ \\
\hline \multirow{2}{*}{$\begin{array}{l}\text { Os processos ajudam a eliminar } \\
\text { desperdícios }\end{array}$} & Sim & $48,3 \%$ \\
\hline & Não & $51,7 \%$ \\
\hline
\end{tabular}

Fonte: dados da pesquisa.

Os dados da tabela mostram que a amostra é heterogênea em diferentes aspectos. Observa-se, também, que a padronização de processos, as diligências e as tarefas se dão pela percepção da maioria dos respondentes por meio do interesse do próprio setor, e que $51,7 \%$ dos respondentes consideram que os processos atuais da PF não ajudam na eliminação de desperdícios. 


\subsection{Análise dos fatores}

Essa etapa do trabalho consiste na realização da AFE para compreender como as variáveis estão relacionadas entre si. Nesse procedimento, foram utilizados todos os itens apresentados na Tabela 3 (com exceção das perguntas de perfil). Os resultados dos testes KMO (valor=0,938) e o teste de esfericidade de Bartlet $(\mathrm{Sig}=0,000)$ foram satisfatórios e indicam a fatorabilidade dos dados.

A seguir, as comunalidades de cada variável foram revisadas e aquelas que apresentam valores menores que 0,5 foram retiradas. Nessa etapa, foram retiradas 5 variáveis das análises.

Quanto à rotação da fatorial, os resultados direcionaram o estabelecimento de 7 fatores, com uma variância explicada total de $66,55 \%$. No entanto, um dos fatores foi composto por apenas uma variável e, por isso, também foi retirado das análises. Na Tabela 5, visualizam-se os fatores, os valores do Alpha de Cronbach de cada fator, as variáveis que os compõem, bem como a carga fatorial de cada variável.

Tabela 5 - Composição dos fatores resultantes da análise fatorial exploratória

\begin{tabular}{|c|c|}
\hline Descrição & Carga fatorial \\
\hline \multicolumn{2}{|l|}{ Fator 01- Maturidade de processos - Alpha de Cronbach = 0,904 } \\
\hline Na Polícia Federal, há pessoas responsáveis pela Gestão de Processos. & 0,775 \\
\hline $\begin{array}{l}\text { Unidades de trabalho que realizam atividades semelhantes (exemplo: outras delegacias ou setores) } \\
\text { usam processos padrão ou similares. }\end{array}$ & 0,773 \\
\hline As habilidades necessárias para executar as tarefas estão definidas e documentadas. & 0,764 \\
\hline Existem programas e atividades de melhoria para manter e aperfeiçoar os processos. & 0,739 \\
\hline São utilizados dados ou informações de desempenho do setor para gerenciar e definir os processos. & 0,722 \\
\hline $\begin{array}{l}\text { No meu setor de trabalho, modelos padrões de processos estão definidos, pelo menos, para cada ativi- } \\
\text { dade principal. }\end{array}$ & 0,677 \\
\hline No meu setor de trabalho, os processos de trabalho estão documentados. & 0,619 \\
\hline \multicolumn{2}{|l|}{ Fator 02- Apoio da liderança - Alpha de Cronbach = 0,909 } \\
\hline $\begin{array}{l}\text { As chefias do meu setor estão constantemente trabalhando para melhorar suas próprias formas de } \\
\text { trabalho, relacionamento e comunicação no setor. }\end{array}$ & 0,862 \\
\hline Meus chefes estão presentes (quando podem) no dia a dia de trabalho. & 0,861 \\
\hline Tenho apoio das chefias do meu setor de trabalho. & 0,817 \\
\hline $\begin{array}{l}\text { Existe uma exigência e um estímulo claro por parte da gestão/chefia de que devo trabalhar com melho- } \\
\text { rias contínuas. }\end{array}$ & 0,816 \\
\hline As chefias do meu setor assumem as responsabilidades por suas ações e decisões. & 0,772 \\
\hline \multicolumn{2}{|l|}{ Fator 03- Melhoria contínua e pensamento a longo prazo- Alpha de Cronbach = 0,855 } \\
\hline Em meu setor, temos tempo para trabalhar na busca de melhorias no nosso trabalho diário. & 0,665 \\
\hline $\begin{array}{l}\text { As decisões e as ações realizadas em nosso setor são baseadas no pensamento a longo prazo, mesmo } \\
\text { tendo que abrir mão de realizar investigações com menor relevância. }\end{array}$ & 0,629 \\
\hline No meu setor, existe uma maneira padronizada de trabalhar em busca de melhorias contínuas. & 0,613 \\
\hline Os processos/diligências auxiliam na motivação dos servidores. & 0,586 \\
\hline $\begin{array}{l}\text { No setor em que trabalho, eu e meus colegas temos uma visão comum de como melhorar as atividades } \\
\text { do setor. }\end{array}$ & 0,578 \\
\hline $\begin{array}{l}\text { Em meu setor, temos um entendimento de que algumas atividades não gerarão retornos e resultados a } \\
\text { curto prazo, somente a longo prazo. }\end{array}$ & 0,535 \\
\hline $\begin{array}{l}\text { Eu e meus colegas nos preocupamos mais em como podemos melhorar as coisas e não em descobrir } \\
\text { quem cometeu um erro. }\end{array}$ & 0,512 \\
\hline \multicolumn{2}{|l|}{ Fator 04- Eliminação de desperdícios - Alpha de Cronbach = 0,865 } \\
\hline Eu sugiro aos outros reduzir os desperdícios em nosso setor de trabalho. & 0,818 \\
\hline Eu sei identificar desperdícios no meu trabalho. & 0,777 \\
\hline Eu procuro reduzir os desperdícios em meu setor de trabalho. & 0,777 \\
\hline $\begin{array}{l}\text { Os desperdícios de qualquer natureza são constantemente "combatidos" por todos em meu setor de } \\
\text { trabalho. }\end{array}$ & 0,709 \\
\hline Em meu setor, eliminar ou diminuir desperdícios é algo com que se trabalha continuamente. & 0,693 \\
\hline \multicolumn{2}{|l|}{$\begin{array}{r}\text { Fator 05- Efetividade de processos }- \text { Alpha de Cronbach }=0,873 \\
\end{array}$} \\
\hline O desempenho dos processos/diligências é confiável. & 0,763 \\
\hline De maneira geral, os processos/diligências são bem efetuados. & 0,75 \\
\hline Os processos/diligências atendem à expectativa dos usuários. & 0,738 \\
\hline Os processos/diligências fornecem resultados de boa qualidade. & 0,716 \\
\hline
\end{tabular}




\begin{tabular}{l|c}
\hline \multicolumn{2}{c}{ Fator 06- Foco nos usuários - Alpha de Cronbach = 0,755 } \\
\hline Eu sei quem são usuários ou investigados ("público-alvo") do meu setor de trabalho ou da PF. & 0,784 \\
\hline Eu sei quais são as necessidades dos “usuários” do meu setor ou da PF e trabalho para atendê-las. & 0,773 \\
\hline Eu sei o que gera valor para os "usuários” do meu setor ou da PF. & 0,744 \\
\hline
\end{tabular}

Fonte: dados da pesquisa.

Ao ser analisada a Tabela 5, pode-se verificar que as questões referentes ao Fator 01, "Maturidade de processos", envolvem elementos como o uso de processos padrão, a definição de responsáveis de processos inteiros, a documentação de processos de trabalho e o suporte de tecnologias de informação (TI). Todos esses elementos dão suporte aos postulados de AlShathry (2016), ao passo que o Alpha de Cronbach foi de 0,904 , indicando alta consistência interna dessas variáveis.

Em relação ao Fator 02, denominado "Apoio da liderança", verifica-se que é formado por cinco variáveis que estão associadas com as chefias dos setores. Para justificar esse fator, pode-se tomar como base a definição de apoio da liderança dada por Siedel et al. (2019), que menciona que a liderança inclui fatores como ter uma visão clara, responsabilidade e vontade de apoiar a iniciativa Lean.

Verifica-se que o Fator 03, "Melhoria contínua e pensamento a longo prazo" foi composto por sete variáveis que relatam sobre melhorias que podem ser efetuadas no ambiente de trabalho e nas atividades diárias dos servidores. Além disso, esse fator engloba as questões que possuem enfoque em práticas que visam resultados a longo prazo. Para Ingelsson e Mårtensson (2014), a melhoria contínua é um valor central do Lean e as organizações obtêm, ao longo do tempo, resultados consideráveis de economia de recursos por meio dessa prática. A agrupação das variáveis de melhoria contínua e pensamento a longo prazo pode ser compreendida ao passo que, de maneira geral, a obtenção de resultados obtidos por meio de práticas de melhoria contínua nem sempre é visualizada a curto prazo.

O Fator 05, nomeado de "Eliminação de desperdícios", possui cinco variáveis que descrevem sobre os desperdícios, tanto nas atividades do trabalho em geral, como, também, nos setores específicos. As práticas envolvendo a redução e a eliminação de desperdícios são outro ponto central da filosofia Lean. 0 foco constante em eliminar desperdícios, visando produtos com maior qualidade ao menor custo, é um valor primário desse sistema de gestão (Scherrer-Rathje et al., 2009).

Quanto ao fator "Efetividade de processos", Fator 04, pode-se verificar que o conjunto de questões permitiu obter um Alpha de Cronbach satisfatório $(0,912)$. As variáveis que compõem esse fator dizem respeito à confiabilidade dos processos, sua qualidade e sua execução e o quanto atendem às expectativas dos usuários. Esses elementos permitem obter um parâmetro sobre a realização dos processos e suas saídas sob diferentes "prismas". Piercy e Rich (2015) argumentam que muitas atividades organizacionais estão sendo vistas e avaliadas apenas como um kit de ferramentas, sem levar em conta todo o processo.

A denominação do Fator 06, "Foco nos usuários", se deve ao fato de ele ser composto por três variáveis que envolvem, em seu conteúdo, o foco dado pelos servidores da PF nos usuários finais, sejam eles internos ou externos. Para Shamah (2013), o objetivo do foco no usuário é que a perspectiva de valor para o público-alvo/clientes jamais deve ser desconsiderada dentro das organizações. Sendo assim, é essencial fornecer produtos e serviços conforme a necessidade do público-alvo/clientes no momento certo e com um preço apropriado para eles.

\subsection{Análise da efetividade e maturidade de processos}

De maneira a avaliar a associação entre a percepção sobre os valores Lean e os fatores de maturidade e de efetividade de processos, foi realizada a correlação de Pearson. Os resultados estão demostrados na Tabela 6 e mostram que quase todas as correlações efetuadas são significativas a 1\%; a exceção é a correlação entre a "Eliminação de desperdícios" e a efetividade de processos (significativa a $5 \%$ ). Observa-se, também, que as correlações desse fator são negativas. Segundo as convenções de Pestana e Gageiro (2008), a única associação que pode ser considerada alta $(>0,7)$ é entre "Melhoria contínua e pensamento a longo prazo" com a maturidade de processos. Todas as demais associações, apesar de significativas, são consideradas moderadas, baixas ou muito baixas. Considerando a hipótese 1 desta pesquisa, pode-se dizer que, para os constructos "Melhoria contínua e pensamento a longo prazo", "Apoio da liderança" e "Foco nos usuários", existe uma associação positiva com a maturidade e a efetividade de processos. Para o fator "Eliminação de desperdícios", essa hipótese não se confirma, 
pois, apesar de muito baixa, a correlação é negativa.

Tabela 6 - Correlação dos valores Lean com a maturidade e a efetividade de processos

\begin{tabular}{l|c|c|c|c}
\hline Fatores & $\begin{array}{l}\text { Melhoria contínua e pensa- } \\
\text { mento a longo prazo }\end{array}$ & $\begin{array}{l}\text { Apoio da lideran- } \\
\text { ça }\end{array}$ & $\begin{array}{l}\text { Eliminação } \\
\text { desperdícios }\end{array}$ & Foco nos usuários \\
\hline Maturidade de processos & $0,735^{* *}$ & $0,082^{* *}$ & $-0,136^{* *}$ & $0,292^{* *}$ \\
\hline Efetividade de processos & $0,594^{* *}$ & $0,097^{* *}$ & $-0,047^{*}$ & $0,436^{* *}$ \\
\hline
\end{tabular}

Fonte: dados da pesquisa.

Nota: ${ }^{* *} \mathrm{e}^{*}$ indicam, respectivamente, correlação significativa a $1 \%$ e $5 \%$.

Para analisar o "comportamento" dos valores Lean Management apresentados no presente trabalho em relação à efetividade e à maturidade de processos, realizou-se, primeiramente, a divisão da amostra em grupos. Para tanto, foi realizada a análise de cluster com base na média das respostas das variáveis dos constructos efetividade e maturidade de processos. Os resultados indicaram como adequada a formação de dois clusters. A Tabela 7 apresenta as estatísticas descritivas dos construtos padronizados conforme a distribuição dos dois clusters formados.

Tabela 7 - Estatísticas descritivas dos clusters maturidade e efetividade de processos

\begin{tabular}{c|c|c|c|c|c|c|c}
\hline \multirow{2}{*}{\begin{tabular}{c} 
Constructo \\
\cline { 2 - 8 }
\end{tabular}} & \multicolumn{3}{|c|}{ Cluster 1 (n=927) } & \multicolumn{3}{c|}{ Cluster 2 (n=76) } & Teste t \\
\hline $\begin{array}{c}\text { Maturidade de proces- } \\
\text { sos }\end{array}$ & 7,97 & 8,00 & 0,72 & 4,47 & 4,86 & 1,48 & 0,000 \\
\hline Efetividade de processos & 8,03 & 8,00 & 0,75 & 5,88 & 6,25 & 2,15 & 0,000 \\
\hline
\end{tabular}

Fonte: dados da pesquisa.

Ao analisar as estatísticas dos construtos que formam cada um dos clusters, observa-se que ambos são estatisticamente diferentes $(\mathrm{Sig}<0,05)$. As análises das estatísticas descritivas mostram que $o$ cluster 1 apresenta altas médias, tanto de maturidade quanto de efetividade de processos. Por outro lado, pode-se verificar que o cluster 2 engloba as observações que apresentam baixas médias, tanto de maturidade quanto de efetividade de processos.

Em face desses clusters, realizou-se o teste t para amostras independentes para avaliar o quanto a percepção de cada um dos valores Lean é estatisticamente diferente em cada um dos clusters. Como o tamanho de cada um dos clusters foi bastante diferente (cluster $1=927$; cluster $2=76$ ), foi realizado, adicionalmente, o teste não paramétrico Mann-Whitney para verificar possíveis resultados diferentes devido ao tamanho dos clusters. Os resultados dos testes estão apresentados na Tabela 8.

Tabela 8 - Teste t para os clusters segundo os valores Lean

\begin{tabular}{l|c|c|c|c|c|c|c|c}
\hline \multirow{2}{*}{ Valores Lean Management } & \multicolumn{2}{|c|}{ Cluster 1 } & \multicolumn{2}{c|}{ Cluster 2 } & \multicolumn{2}{c|}{ Teste t } & \multicolumn{2}{c}{ Mann-Whitney } \\
\cline { 2 - 9 } & Média & Desvio & Média & Desvio & Valor & Sig & Valor & Sig \\
\hline $\begin{array}{l}\text { Melhoria contínua e pensamen- } \\
\text { to a longo prazo }\end{array}$ & 7,99 & 0,67 & 5,43 & 1,74 & 12,753 & 0,000 & 5377,0 & 0,000 \\
\hline Apoio da liderança & 5,98 & 1,56 & 5,46 & 2,43 & 1,858 & 0,067 & 30745,0 & 0,065 \\
\hline Eliminação de desperdícios & 5,98 & 1,53 & 6,73 & 1,38 & $-4,122$ & 0,000 & 25468,0 & 0,000 \\
\hline Foco nos usuários & 8,06 & 0,84 & 7,71 & 1,86 & 1,632 & 0,107 & 34153,0 & 0,656 \\
\hline
\end{tabular}

Fonte: dados da pesquisa.

Pela análise da Tabela 8, nota-se, primeiramente, que os resultados dos dois testes não divergiram quanto aos fatores em que há diferença significativa das médias $(\operatorname{sig}<0,05)$. Os fatores "Melhoria contínua e pensamento a longo prazo" e "Eliminação de desperdícios" apresentaram diferenças de médias significativas para os dois grupos analisados. Especificamente, pode-se verificar que a média da percepção quanto à "Melhoria contínua e pensamento a longo prazo" é maior no cluster 1, o qual apresenta valores mais elevados de efetividade e de maturidade de processos. Isso significa que o grupo com melhores percepções de maturidade e de efetividade de processos apresenta, também, uma melhor avaliação desse valor Lean em específico (isso vai ao encontro da hipótese 2). 0 mesmo não se aplica para o fator "Eliminação de desperdícios", em que a média é mais baixa no cluster 1. Nessa situação, o grupo com pior percepção de maturidade e de efetividade de processos é que apresenta, em média, melhores percepções desse fator (o que vai de encontro à hipótese 2). Por fim, pode-se verificar que não houve diferença estatisticamente significante entre os dois clusters para os fatores "Apoio da liderança" e "Foco nos usuários".

Para finalizar a análise da maturidade e de efetividade de processos, procurou-se verificar em que medida há diferença na percepção de efetividade de processos considerando a maturidade. Para 
tanto, foi realizada uma nova análise de cluster somente com as questões que compõem o fator maturidade de processos. Os resultados indicaram como adequada a formação de dois clusters (Tabela 9).

Tabela 9 - Estatísticas descritivas dos clusters maturidade de processos

\begin{tabular}{c|c|c|c|c|c|c|c}
\hline \multirow{2}{*}{$\begin{array}{c}\text { Questões do fator } \\
\text { maturidade }\end{array}$} & \multicolumn{3}{|c|}{$\begin{array}{c}\text { Cluster 1 (n=928) } \\
\text { Maturidade alta }\end{array}$} & \multicolumn{3}{c|}{$\begin{array}{c}\text { Cluster 2 (n=75) } \\
\text { Maturidade baixa }\end{array}$} & Teste t \\
\cline { 2 - 8 } & Média & Mediana & $\begin{array}{c}\text { Desvio Pa- } \\
\text { drão }\end{array}$ & Média & Mediana & $\begin{array}{c}\text { Desvio Pa- } \\
\text { drão }\end{array}$ & \begin{tabular}{c} 
Sig \\
\hline $1^{*}$
\end{tabular} \\
\hline $2^{*}$ & 7,94 & 9,00 & 1,02 & 4,16 & 4,00 & 2,11 & 0,000 \\
\hline $3^{*}$ & 7,92 & 8,00 & 1,14 & 4,69 & 5,00 & 2,22 & 0,000 \\
\hline $4^{*}$ & 7,90 & 8,00 & 1,06 & 4,09 & 4,00 & 2,14 & 0,000 \\
\hline $5^{*}$ & 8,00 & 8,00 & 1,10 & 3,49 & 3,00 & 1,94 & 0,000 \\
\hline $6^{*}$ & 8,06 & 8,00 & 1,04 & 3,41 & 3,00 & 2,05 & 0,000 \\
\hline $7^{*}$ & 7,99 & 8,00 & 1,16 & 5,60 & 6,00 & 2,50 & 0,000 \\
\hline
\end{tabular}

Fonte: Dados da pesquisa.

Nota: *a ordem dessas questões é a mesma que apresentada na Tabela 4.

Pode-se verificar que o cluster 1 apresenta as maiores médias e medianas para as questões sobre maturidade, o que pressupõem um cluster com alta maturidade de processos. Por outro lado, pode-se verificar que o cluster 2 engloba as observações que apresentam baixas médias e medianas em todas as questões analisadas, o que indica um cluster com baixa maturidade de processos. Deve-se ressaltar que todas as variáveis observadas contribuem para formar os clusters $($ Sig $<0,05)$.

A partir da identificação desses clusters, foram realizados os testes t e Mann-Whitney para avaliar se a efetividade de processos é estatisticamente diferente nos clusters de maturidade de processos. Especificamente, a intenção é avaliar se o cluster com maior maturidade apresenta realmente maior efetividade. Os resultados desse teste estão apresentados na Tabela 10.

Tabela 10 - Teste t para efetividade de processos X os clusters de maturidade

\begin{tabular}{c|c|c|c|c|c|c|c|c}
\multirow{2}{*}{ FATOR } & \multicolumn{2}{|c|}{$\begin{array}{c}\text { Cluster } 1 \\
\text { Maturidade alta }\end{array}$} & \multicolumn{2}{c|}{$\begin{array}{c}\text { Cluster } 2 \\
\text { Maturidade baixa }\end{array}$} & \multicolumn{2}{c}{ Mann-Whitney } \\
& Média & Desvio Padrão & Média & Desvio Padrão & Valor & Sig & Valor & Sig \\
\hline EFETIVIDADE & 8,01 & 0,78 & 6,11 & 2,27 & 75,41 & 0,000 & 15073,5 & 0,000 \\
\hline
\end{tabular}

Fonte: dados da pesquisa.

Os resultados expostos na Tabela 10 permitem verificar que o cluster 1, caracterizado pelo alto nível de maturidade de processos, é o que apresenta a maior média de efetividade de processos, que é estatisticamente diferente (teste t e teste Mann-Whitney) da média do cluster 2. Isso permite confirmar a hipótese 3 desta pesquisa: o grupo com melhor percepção de maturidade de processos também apresenta, em média, melhores percepções quanto à efetividade de processos.

\section{Discussão dos resultados}

Os resultados do presente estudo indicam valores Lean que possuem uma associação com a maturidade e a efetividade de processos. 0 primeiro é a "Melhoria contínua e o pensamento a longo prazo", o que corrobora Rymaszewska (2017). A noção a longo prazo, ao passo que é um valor basilar da filosofia de gestão Lean (Liker, 2004), é essencial no gerenciamento de processos na medida em que envolve a noção de melhorias padronizadas ao longo do tempo. Isso requer a incorporação de um foco organizacional voltado para melhorias contínuas que, podem não gerar resultados imediatos a curto prazo, mas efetivam a geração de pequenos resultados que contribuem para o alcance de resultados maiores e a geração de valor. Esse foi o único fator que apresentou uma avaliação significativa maior no grupo com maior percepção de maturidade e de efetividade de processos.

Os fatores "Apoio da liderança" e "Foco no público-alvo" também apresentaram uma associação positiva com as medidas de processo analisadas. Ambos os valores estão, em teoria, associados, uma vez que o suporte da liderança é o estímulo primordial para a execução de ações e de transformações em atividades operacionais diárias. Alefari, Salonitis e Xu (2017) explicam que os líderes de uma organização possuem, entre outros papéis, o de viabilizar e oferecer caminhos para a melhoria do desempenho do colaborador e de suas atividades. Ter envolvimento dos colaboradores e suporte da liderança no aprimoramento das atividades é essencial (Lu, Laux \& Antony, 2017), mas ainda um desafio para as muitas organizações (Tarhan et al., 2016), pois estas necessitam da geração de resultados. Isso acaba direta ou indiretamente repercutindo na necessidade de maturidade e de 
efetividade de processsos.

$\mathrm{Na}$ prática, conhecer elementos que sirvam de base para as melhorias incrementais em processos (como os valores Lean) repercutem em outputs organizacionais melhores. A PF pode se beneficiar e melhorar a prestação de seus serviços na medida em que tornar a prospecção e a aplicação dos valores Lean uma constante nos departamentos e em suas unidades organizacionais. Ao passo que a abordagem de processos fornece uma estrutura ampla para as melhorias em procedimentos organizacionais, o Lean é uma filosofia ampla subjacente à melhoria de processos, com foco na eliminação de desperdícios e na maximização de valor (Dave, 2017).

Outro fator que demostrou uma diferença de médias significativa para os clusters de alta $v s$ baixa maturidade e efetividade de processos é a "Eliminação de desperdícios". No entanto, o que chama a atenção nesse resultado é o fato de a média no cluster 1 (alta maturidade e alta efetividade) ser menor do que para o cluster 2 . Isso significa que, em média, aquele grupo que percebe melhor efetividade e maturidade nos processos não necessariamente percebe e trabalha de maneira a identificar, diminuir e eliminar os desperdícios. Esse fato diverge da hipótese 2 delineada neste artigo. Diferentes fatos podem explicar esse resultado como, por exemplo, a não compreensão do que seja um desperdício segundo a filosofia Lean ou o fato de os respondentes não perceberem uma determinada ação ou atitude como um desperdício. As rotinas organizacionais levam os servidores a executar os processos sem a percepção de que há desperdícios envolvidos e esta é uma oportunidade de melhoria e reflexão para as instituições públicas.

As características específicas de instituições públicas podem, também, explicar o fato supracitado. Apesar de ter ocorrido a reforma pública gerencial que visa a descentralização administrativa, ações voltadas ao público, a orientação para resultados, a maior confiança nos gestores públicos e o incentivo à criatividade e à inovação, etc. (Bresser-Pereira \& Spink, 2006), a mudança para uma nova forma gerencial pode não ser tão rápida. 0 "velho modelo" de gestão, caracterizado como burocrático e com o controle rígido de processos (Ribeiro \& Mancedo, 2013), necessita da inserção de práticas e de melhorias (como as do sistema Lean) para a efetivação das mudanças. Além disso, é necessário que essas mudanças estejam alinhadas com o planejamento e as diretrizes contratuais vigentes (Mizael, Chagas \& Antonialli, 2020).

Os resultados deste estudo também corroboram para a percepção de que processos com maior maturidade possuem mais efetividade. A maturidade dos processos reflete em que medida a organização obtém sucesso em sistematicamente aumentar as capabilities da organização e de seus próprios processos, a fim de oferecer maior desempenho ao longo do tempo (Isik, Mertens \& Van den Bergh, 2013). A noção de eficiência e de eficácia na gestão pública não deve ser atrelada somente à agilidade e à rapidez do serviço prestado. A esses, devem ser vinculados elementos como qualidade, expectativa dos usuários, confiabilidade e baixo custo analisados deste trabalho. Para isso, a efetividade pode ser envolvida como medida paralela à maturidade para verificar os resultados dos processos. Schymik et al. (2007) já haviam endereçado a utilização de medidas de maturidade e de efetividade de processo como complementares e úteis para avaliação de processos. A maturidade de processos serve como parâmetro à organização para definir padrões básicos comuns a fim de garantir a eficiência e a efetividade de processos.

Szelagowski e Berniak-Woźny (2020) destacam que não se pode criar valor em uma organização sem alinhamento das atividades com a estratégia de negócios da organização. Ademais, Araújo e Lemos (2020) destacam em seu estudo uma certa carência de padronização dos processos em procedimentos públicos (no caso deles, os licitatórios), o que dificulta o gerenciamento. Portanto, os elementos de maturidade de processos avaliados neste trabalho (como o quanto a organização utiliza dados ou informações de desempenho do setor, o quanto ela avalia as habilidades necessárias e padroniza os procedimentos e o suporte de TI, etc.) podem ser inputs relevantes para melhorias contínuas e para a efetividade de processos na instituição estudada.

\section{Considerações Finais}

No Brasil, o setor público vem sofrendo com a escassez de recursos, principalmente financeiros, há alguns anos. Revisar prioridades, melhorar o atendimento ao público, a qualidade dos serviços e reduzir gastos desnecessários estão entre algumas das cobranças constantes da sociedade. Diante disso, tanto a governança quanto a gestão pública necessitam de ferramentas e de práticas que permitam a reorganização das atividades administrativas para obtenção de melhores resultados e 
melhor eficiência. Tomando em consideração esses apontamentos, este trabalho teve como questão de pesquisa verificar "em que medida os valores Lean se associam e variam diante das medidas de efetividade e de maturidade de processos?". Alinhado a esse problema, o objetivo foi o de avaliar os valores Lean e sua relação e variação com a maturidade e a efetividade da Gestão de Processos.

Para responder essa pergunta de pesquisa e alcançar esse objetivo, realizou-se uma pesquisa survey com os servidores ativos da PF do Brasil. Os dados obtidos foram analisados, primariamente, por meio da técnica da AFE, correlação de Pearson, análise de cluster e testes t. Os resultados desta pesquisa mostraram que a média do fator "Melhoria contínua e pensamento a longo prazo" foi significativamente maior no cluster em que a maturidade e a efetividade de processos possuíam maiores médias. 0 resultado oposto pode ser verificado com o fator "Redução de desperdícios". Além disso, outra conclusão que os resultados deste estudo permitem auferir é que quanto maior a maturidade de processos, maior é a efetividade dos mesmos.

Diante do exposto, este estudo se diferencia dos demais na medida em que proporciona uma avaliação sobre valores Lean e sua relação com a maturidade e a efetividade de processos, bem como testa diferenças de média desses valores em dois grupos distintos. Esse avanço na literatura sobre o assunto proporciona um direcionamento sobre a usabilidade de valores da filosofia Lean para fortalecimento de uma cultura de mudança e de melhoria da Gestão de Processos. A utilização desses valores em consonância às ferramentas - como o mapeamento de processos, o mapeamento de fluxo de valor, "os 5 porquês" e o root cause analysis - pode gerar diretrizes para gerenciar as atividades de melhoria de processo (Dave, 2017).

Além disso, o presente estudo consolida medidas de efetividade de processos como complementares à maturidade de processos. No entanto, as especificidades do setor público devem ser tomadas para o conjunto de mudanças e de decisões, pois a reforma gerencial não deve ser a mera transferência de tecnologias gerencias do privado para o público (Siqueira \& Mendes, 2009). Os resultados desta pesquisa não se limitam às empresas ou às instituições que já utilizam ou praticam a abordagem de processos. As estruturas organizacionais voltadas à tradicional divisão funcional também podem usufruir dos resultados deste trabalho à medida que incorporarem, em suas rotinas e, gradativamente, em sua cultura, os valores Lean aqui estudados.

A realização deste estudo possui, dentre suas principais limitações, a não aleatoriedade dos dados e a forma como a coleta de dados foi realizada. Isso por que, ao passo que uma das formas de divulgação da pesquisa foi feita por meio de grupos de whatsapp, os indivíduos que não utilizam essa ferramenta de comunicação ou que estavam fora dos grupos podem não ter sido incentivados a participar da mesma forma (mesmo que tivessem recebido um e-mail convite). Outra limitação desta pesquisa foi a falta de uma possibilidade de explicar com mais detalhes os tipos de desperdício relacionados e analisados na gestão Lean. Talvez esse tenha sido o principal problema de haver uma correlação negativa do constructo "Eliminação de desperdícios" e a maturidade e a efetividade de processos.

Estudos futuros podem avaliar as medidas de valores Lean, maturidade e efetividade de processos para realizar pesquisas que visem demonstrar relações de impacto entre esses fatores, por exemplo, por meio da análise de regressão. Utilizar técnicas como análise de regressão e a modelagem de equações estruturais para avaliar como valores Lean afetam parâmetros da gestão de processos pode gerar insights valiosos para essa temática. Além disso, podem ser utilizadas outras medidas para avaliar essas relações, como medidas de desempenho organizacional e operacional e as práticas do Lean Management. Por fim, outra oportunidade de pesquisa é a análise específica dos desperdícios no serviço público e a projeção de maneiras de reduzi-los. 0 entendimento dos desperdícios pode ser um dos principais caminhos de otimizar as atividades operacionais, a partir da eliminação gradual destes, por meio de pequenas mudanças no dia a dia dos colaboradores.

\section{Referências}

Aka, A., Isah, A. D., Eze, C. J., \& Timileyin, O. (2019). Application of lean manufacturing tools and techniques for waste reduction in Nigerian bricks production process. Engineering, Construction and Architectural Management., 27(3), 658-679.

Alefari, M., Salonitis, K., \& Xu, Y. (2017). The role of leadership in implementing lean manufacturing. Procedia CIRP, 63, 756-761.

AlShathry, O. (2016). Business process management: a maturity assessment of Saudi Arabian organizations. Business Process Management Journal, 22(3), 507-521. 
Andrade, E.; Rasoto, V. I., \& Carvalho, H. A. (2018) Gerenciamento de processos nas Instituições Federais de ensino superior Brasileiras. RBPD - Revista Brasileira de Planejamento e Desenvolvimento, $7(2), 171-201$.

Antony, J., Rodgers, B. \& Gijo, E. V. (2016). Can Lean Six Sigma make UK public sector organisations more efficient and effective? International Journal of Productivity and Performance Management, 65(7), 995-1002.

Aqlan, F., \& Al-Fandi, L. (2018). Prioritizing process improvement initiatives in manufacturing environments. International Journal of Production Economics, 196, 261-268.

Araújo, G. B. P., \& Lemos, L. B. S. (2020). A Gestão de Compras Públicas: um Estudo de Caso da Central de Compras do Distrito Federal. TPA - Teoria e Prática Em Administração, 10(2), 124-137.

Bhasin, S., \& Burcher, P. (2006). Lean viewed as a philosophy. Journal of Manufacturing Technology Management, 17(1), 56-72.

Carballo-Mendivil, B., Arellano-Gonzalez, A., \& Rios-Vazquez, N. J. (2018). Lean processes management as a principle of improvement: a case applied to a commerce. 3C Empresa, 7(3), 61-80.

Castro, B. K. A., Dresch, A., \& Veit, D. R. (2020). Key critical success factors of BPM implementation: a theoretical and practical view. Business Process Management Journal. 26(1), 239-256.

Cavdur, F., Yagmahan, B., Oguzcan, E., Arslan, N., \& Sahan, N. (2019). Lean service system design: a simulation-based VSM case study. Business Process Management Journal, 25(7), 1802-1821.

Dave, B. (2017). Business process management-a construction case study. Construction Innovation, 17(1), 50-67.

Duarte, S., \& Machado, V. C. (2017). Green and lean implementation: an assessment in the automotive industry. International Journal of Lean Six Sigma, 8(1), 65-88.

Dumas, M.,La Rosa, J., Mendling M., \& Reijers, H. A. (2013). Introduction to business process management. Fundamentals of Business Process Management. Springer Berlin Heidelberg, 1(2), 1-31.

Ferrari, A., Witschel, H., Spagnolo, G. \& Gnesi, S. (2018). Improving the quality of business process descriptions of public administrations: Resources and research challenges. Business Process Management Journal, 24(1), 49-66.

Ghobakhloo, M., Fathi, M., Fontes, D.B.M.M., \& Ching, N. T. (2018). Modeling lean manufacturing success. Journal of Modelling in Management, 13(4), 908-931.

Gómez-Luciano, C. A., Domínguez, F. R. R., González-Andrés, F., \& De Meneses, B. U. L. (2018). Sustainable supply chain management: Contributions of supplies markets. Journal of Cleaner Production, 184, 311-320.

Gudem, M., Steinert, M., Welo, T., \& Leifer, L. (2013). Redefining customer value in lean product development design projects. Journal of Engineering, Design and Technology, 11(1), 71-89.

Gupta, S., Sharma, M. \& Sunder, M.V. (2016). Lean services: a systematic review. International Journal of Productivity and Performance Management, 65(8), 1025-1056.

Hair, J. F., Black, W. C., Babin, B. J., Anderson, R. E., \& Tatham, R. L. (2005). Análise multivariada de dados. Porto Alegre - POA: Bookman Editora.

Hernaus, T., Vuksic, V. B., \& Štemberger, M. I. (2016). How to go from strategy to results? Institutionalising BPM governance within organisations. Business Process Management Journal, 22(1), 173-195.

Hussain, M., \& Malik, M. (2016). Prioritizing lean management practices in public and private hospitals. Journal of Health Organization and Management, 30(3), 457-474.

Ingelsson, P., \& Mårtensson, A. (2014). Measuring the importance and practices of Lean values. The TQM Journal, 26, 463-474.

Işik, Ö., Mertens, W., \& Van den Bergh, J. (2013). Practices of knowledge intensive process management: quantitative insights. Business Process Management Journal, 19(3), 515-534.

Johansson, P. E., \& Osterman, C. (2017). Conceptions and operational use of value and waste in lean manufacturing-an interpretivist approach. International Journal of Production Research,55(23), 6903-6915.

Klun, M., \& Trkman, P. (2018). Business process management - at the crossroads. Business Process Management Journal, 24(3), 786-813.

Kohlbacher, M. (2010). The effects of process orientation: A literature review. Business Process Management Journal, 16(2), 135-152.

Krukowski, K., \& Raczyńska, M. (2019). Attributes of Process Maturity of Public Administration Units in Poland. Administrative Sciences, 9(4), 84. 
Liker J.K. (2004). The Toyota Way - 14 Management Principles from the World's Greatest Manufacturer. New York: NY: McGraw-Hill.

Lu, J., Laux, C., \& Antony, J. (2017). Lean Six Sigma leadership in higher education institutions. International Journal of Productivity and Performance Management, 66(5), 638-650.

Madsen, D. Ø., Berg, T., Stenheim, T., Moum, J. V., Bordewich, I. O., \& Storsveen, M. (2019). The longterm sustainability of lean as a management practice: Survey evidence on diffusion and use of the concept in Norway in the period 2015-2017. Sustainability, 11(11), 3120.

Maldonado, M. U., Leusin, M. E., Bernardes, T. C. A., \& Vaz, C. R. (2020). Similarities and differences between business process management and lean management. Business Process Management Journal. Vol. ahead-of-print No. ahead-of-print.

Marodin, G.A., Tortorella, G.L., \& Godinho Filho, M. (2017). The moderating effect of Lean supply chain management on the impact of Lean shop floor practices on quality and inventory. Supply Chain Management, 22(6), 473-485.

Mizael, G. A., Chagas, C. G., \& Antonialli, L. M. (2020). Perspectivas da Terceirização na Administração Pública na Nova Abordagem de Contratação. TPA - Teoria e Prática em Administração, 10(1), 25-37.

Modig, N., \& Åhlström, P. (2012). This is lean: resolving the efficiency paradox. Stockholm: Rheologica publishing.

Narayanamurthy, G., \& Gurumurthy, A. (2016). Systemic leanness - an index for facilitating continuous improvement of lean implementation. Journal of Manufacturing Technology Management, 27(8), 1014-1053.

Oliveira, D. P. R. de. (1994). Sistemas, organização e métodos: uma abordagem gerencial. São Paulo SP: Atlas.

Pestana, M. H., \& Gageiro, J. N. (2008). Análise de dados para ciências sociais: a complementaridade do SPSS. Lisboa: EdiçõesSílabo, Lda.

Piercy, N., \& Rich, N. (2015). The relationship between lean operations and sustainable operations. International Journal of Operations \& Production Management, 35(2), 282-315.

Polícia Federal do Brasil. (2014). Portaria no 4453/2014-DG/DPF, de 16 de maio de 2014. Recuperado de http://www.pf.gov.br/institucional/planejamento-estrategico.

Rentes, V. C., de Pádua, S. I. D., Coelho, E. B., Cintra, M. A. D. C. T., Ilana, G. G. F. \& Rozenfeld, H. (2019)”, Implementation of a strategic planning process oriented towards promoting business process management (BPM) at a clinical research centre (CRC)", Business Process Management Journal, 25(1), 707-737.

Rymaszewska, A. (2017). Lean implementation and a process approach-an exploratory study. Benchmarking: An International Journal, 24(5), 1122-1137.

Scherrer-Rathje, M., Boyle, T. A., \& Deflorin, P. (2009). Lean, take two! Reflections from the second attempt at lean implementation. Business horizons, 52(1), 79-88.

Schymik, G., Kulkarni, U., \& Freeze, R. (2007, December). Impact of knowledge management systems on knowledge intensive business processes. In: AMCIS (Americas Conference on Information Systems), Proceedings, CA.

Shah, R., \& Ward, P. T. (2003). Lean manufacturing: context, practice bundles, and performance. Journal of operations management, 21(2), 129-149.

Shah, R., \& Ward, P. T. (2007). Defining and developing measures of lean production. Journal of operations management, 25(4), 785-805.

Shamah, R. A. (2013). A model for applying lean thinking to value creation. International Journal of Lean Six Sigma, 4(2), 204-224.

Siedel, H., Rust, M., Goth, K., Krüger, A., \& Heidenfelder, W. (2019). A candidate for "Global Heritage Stone Resource" designation from Germany. Episodes Journal of International Geoscience, 42(2), 8191.

Siqueira, M. V. S., \& Mendes, A. M. (2009). Gestão de pessoas no setor público e a reprodução do discurso do setor privado. Revista do Serviço Público, 60(3), 241-250.

Szelagowski, M., \& Berniak-Woźny, J. (2020). The adaptation of business process management maturity models to the context of the knowledge economy. Business Process Management Journal.26(1), 212-238

Tarhan, A., Turetken, O., \& Reijers, H. (2016). Business process maturity models: a systematic literature review. Information and Software Technology,75(7), 122-134. 
Uhrin, Á., Bruque-Cámara, S., \& Moyano-Fuentes, J. (2017). Lean production, workforce development and operational performance. Management Decision, 55(1), 103-118.

Van Looy, A., De Backer, M., \& Poels, G. (2011). Defining business process maturity. A journey towards excellence. Total Quality Management \& Business Excellence, 22(11), 1119-1137.

Waterman, J., \& McCue, C. (2012). Lean thinking within public sector purchasing department: the case of the UK public service. Journal of Public Procurement, 12(4), 505-527.

Wickramasinghe, G. L. D., \& Wickramasinghe, V. (2017). Implementation of lean production practices and manufacturing performance. Journal of Manufacturing Technology Management, 28(4), 531-550.

Womack, J. P., Jones, D. T. \& Roos, D. (2004). A máquina que mudou o mundo. Rio de Janiero - RJ: Elsevier.

Zairi, M. (1997). Business process management: a boundaryless approach to modern competitiveness. Business Process Management Journal, 3(1), 64-80. 